\title{
Towards Consumer-Empowering Artificial Intelligence
}

\author{
Giuseppe Contissa ${ }^{1,2}$, Francesca Lagioia ${ }^{2}$, Marco Lippi ${ }^{3}$, Hans-Wolfgang Micklitz ${ }^{2}$ \\ Przemysław Pałka ${ }^{2}$, Giovanni Sartor ${ }^{1,2}$ and Paolo Torroni ${ }^{4}$ \\ ${ }^{1}$ CIRSFID, Alma Mater - Università di Bologna, Italy \\ ${ }^{2}$ Law Department, European University Institute, Florence, Italy \\ ${ }^{3}$ DISMI - Università di Modena e Reggio Emilia, Italy \\ ${ }^{4}$ DISI, Alma Mater - Università di Bologna, Italy \\ marco.lippi@unimore.it, \{giuseppe.contissa, giovanni.sartor, paolo.torroni\}@unibo.it, \\ $\{$ francesca.lagioia, hans.micklitz, przemyslaw.palka\}@eui.eu
}

\begin{abstract}
Artificial Intelligence and Law is undergoing a critical transformation. Traditionally focused on the development of expert systems and on a scholarly effort to develop theories and methods for knowledge representation and reasoning in the legal domain, this discipline is now adapting to a sudden change of scenery. No longer confined to the walls of academia, it has welcomed new actors, such as businesses and companies, who are willing to play a major role and seize new opportunities offered by the same transformational impact that recent AI breakthroughs are having on many other areas. As it happens, commercial interests create new opportunities but they also represent a potential threat to consumers, as the balance of power seems increasingly determined by the availability of data. We believe that while this transformation is still in progress, time is ripe for the next frontier of this field of study, where a new shift of balance may be enabled by tools and services that can be of service not only to businesses but also to consumers and, more generally, the civil society. We call that frontier consumer-empowering AI.
\end{abstract}

\section{Introduction}

The history of Artificial Intelligence (AI) and Law started about four decades ago. For most of its existence, this discipline has been primarily concerned with attempts to formalize legal reasoning and create expert systems. As such, it was confined to the periphery of the mainstream legal scholarship, targeting an audience composed to a great extent by legal theorists and logicians.

This has changed about in the last five years, owing in large part to successful implementations of $\mathrm{AI}$ in numerous fields of commerce. The focus of AI and Law as a discipline has expanded beyond representation and reasoning. On the one hand, a number of legal-tech applications have entered the marketplace, often based on machine-learning and data-driven approaches. On the other hand, policy issues have become prominent, motivating many scholars to address the social and legal challenges posed by the widespread use of AI technology. Such a shift was, in our opinion, not only beneficial but even necessary, dictated by a need to adapt to a changing world. In 2015, Gartner predicted that in three years' time 50 percent of business ethics violations would occur through improper use of big data analytics. ${ }^{1}$ At the same time, we believe that, alongside with addressing the challenges posed by the uses of AI by businesses, interdisciplinary legal-technical research should also focus on harvesting the fruits of AI and bringing them to benefit consumers and civil society. Although this new avenue of research will certainly build upon the results achieved throughout the long-standing history of the discipline, yet the scenarios we envisage will likely pave the way to more focused methodologies, that will have to be adequately characterized in order to be societally relevant.

The pervasive use of AI by businesses poses new types of challenges to consumer law and policy [Helveston, 2015]. The synergy between AI and big data, in fact, enhances the power of producers and intermediaries, and their dominance over consumers. AI systems can use big data to anticipate consumer behaviour and to try and trigger desired reactions. Hence, consumers can be outwitted, manipulated, and induced into suboptimal purchases or other unwitting choices [O'Neil, 2016]. Privacy is affected by these phenomena in multiple ways: consumer data are continuously collected by on-line and off-line consumer behaviour tracking, they are stored and merged with other data sources, and processed in order to elicit further information about consumers

\footnotetext{
${ }^{1}$ https://www.gartner.com/newsroom/id/3144217
} 
though profiling. The resulting indications about consumer attitudes, weaknesses and propensities are deployed in decisions affecting individual consumers, or in attempts to influence their behaviour. Consumers are mostly left in the dark: they cannot be aware of how they are being monitored and manipulated by AI systems. Moreover, they are unable to contest the practices being implemented. Even consumer organizations (collective entities representing consumers' interest) and public authorities have been thus far unable to counter such practices, having no means to rival the resources and technological superiority of the companies that combine $\mathrm{AI}$ and big data, both Internet intermediaries and other large commercial entities (maybe with the exception of national security agencies).

Yet, we should not consider AI to be necessarily only at the service of producers and intermediaries. AI can also be exploited to unlock consumer-empowering technologies, that is those technologies and tools aimed to serve the cause of both individual consumers (apps, browser extensions, enhancing understanding and autonomy) and their organizations (automating oversight and law enforcement process). Such technologies may support the creation of effective consumer counter-power, fortifying the consumers' defences, for instance by protecting them against unfair or even abusive commercial practices.

A number of early studies seem to indicate a growing attention to this cause. However, we are only at the dawn of consumer-empowering AI. Many are the open challenges that still need to be addressed: protecting consumers against unwanted monitoring and data collection, supporting consumers and their organizations in the detection and contesting of unfair uses of AI and more generally of Information and Communication Technologies (ICT), enabling consumers and their organizations to control the fairness of commercial practice, and so on.

This is a great challenge both for AI and for the civil society, as AI technologies are becoming increasingly integrated in our lives of citizens. Among the challenges that Wagstaff [2012] identifies in order for machine learning to make an impact on society in the near future there is one that stands out: it is the passing of a law, or the making of a legal decision, that relies on the result of a machine learning analysis. Although legal decisions solely ruled by automatic systems are prohibited by some jurisdictions [Article 29 Working Party, 2017], we have no doubt that such a scenario will become plausible in the close future, and that intelligent systems able to support citizens and administrations within the legal domain will become available to the community.

This article offers a critical discussion of the evolution of the field of AI and Law under this perspective, starting from the early days, until the key challenges addressed by today's research, and proposes consumer-empowering AI as the next frontier of this important discipline.

\section{The Past: AI and Law from Knowledge Modeling to Machine Learning}

Until a decade ago, both the legal reflections on AI and the development of AI models for the law had been mostly inspired by the paradigm of logical inference and knowledge representation [Gordon et al., 2009]. Only a few contributions were devoted to the legal aspects of AI. They were mainly about issues such as the legal protection of knowledge bases, or the regulation of AI agents (contract and torts). These academic contributions did not address significant issues in the legal practices, since most applications were limited to toy examples. Even the successful projects, such as larger expert systems for public administrations, did not raise significantly new legal problems.

The social and legal issues raised by AI systems developed at that time were not different from those raised by equivalent non-AI software. For instance, the issues consumers would face when interacting with an on-line shopping system were not unlike those they would face when interacting with a rulebased system. Since both systems would operate on the basis of pre-coded knowledge, from the legal perspective, they would raise the same legal issues, and be regulated accordingly. Some case studies dealt with harms caused by faulty software, or the validity of a contract including an unwanted term because of a software error [Sartor, 2009]. Legal doctrine also addressed some AI-related issues [Allen and Widdison, 1996], but few relevant cases could be found in legal practice.

Research on AI applications in the legal domain started in the '70s and produced a number of models for representing legal knowledge and reasoning, namely rule-based, argument-based, and case-based models (for an overview, see [Prakken and Sartor, 2015]).

According to rule-based models, the law is viewed as an axiomatic system, namely as a set of premises from which legal conclusions could be achieved through logical inference [Allen and Saxon, 1994]. Once a legal text and a body of facts have been clearly represented in a formal language, the legal conclusions would follow from that representation as a matter of deductive or defeasible inference. This enables using logical formalisms for the analysis of concepts (ontologies) and clear drafting of rules, possibly including nonmonotonic techniques for representing rule-exception structures and legal hierarchies [Gordon et al., 2009].

Argument-based approaches stem from the consideration that legal reasoning goes beyond the literal meaning of the legal rules and involves appeals to precedents, principles, policies and purposes. Legal rules are not just applied but are themselves the subject of reasoning, as in legal interpretation. But facts may also be the subject of legal reasoning, that is reasoning about how a fact can be proven to be true or false. Such reasoning is carried out in the form of legal argumentation. A formal model of legal argumentation provides for the representation of arguments and their relations, and for the specification of their semantics. Researchers in $\mathrm{AI}$ and Law have produced a variety of computable models of legal argumentation [Verheij, 2003; Gordon et al., 2007; Prakken, 2010] that widely differ for such characteristics.

Case-based models represent legal cases so that a computer program can reason over cases by analogy. In particular, they provide methods to compare a new case with past cases, select the most relevant among them, and generate legal arguments by for and against a conclusion in a 
new case. A legal case has various aspects to be represented (fact, concepts, factors) each with its own modes of reasoning: determining the facts, classifying the facts under legal concepts or conditions, and deriving legal consequences from the classified facts [Ashley, 1991; Prakken and Sartor, 1998; Horty and Bench-Capon, 2012; Prakken et al., 2015].

However, it must be noted that research in AI \& Law has mainly focused on theoretical aspects and has seldom reached the marketplace. In fact, several systems have been developed according to one of the three models mentioned above, or a combination thereof, but they have had limited practical applications, with the exception of several rule-based systems. One of the few successful commercial products of this type is a system developed in Australia, originally named Softlaw, which was later renamed Ruleburst and eventually became Oracle's Policy Automation system. The system, used in many applications for the Public Administration in Australia, UK and US, includes a set of tools for building knowledgebases of regulations, for checking their correctness and consistency and for using them interactively.

Some attempts have been done also in the domain of consumer law. A prominent example is the Latent Damage System [Capper and Susskind, 1988]. Yet, such systems did not have a significant impact on legal practice. The main reason boils down to a general problem of knowledge-based systems, known as the knowledge representation bottleneck. The legal knowledge to be employed by the computational models of such systems, in fact, had to be manually extracted from legal sources, including statutes, regulations, cases, contracts, and other texts. Thus, human experts had to read the legal texts and represent relevant parts thereof in a form that could be interpretable for computational models. Such a process was slow and labor-intensive. The same problem emerged when the knowledge had to be revised, for example when new laws had to be added to the knowledge base, or abrogated laws had to be removed. Consumer law particularly suffered from having to deal with heterogeneous and dynamic material, including contracts, policies, and unstructured textual sources with different legal value.

Research in AI and Law has evolved in the last years, giving rise to a number of applications of machine learning and text analytics to the legal domain. These include: (1) legal text analytics for identifying and representing legal concepts, to define kinds of annotations, concepts, and relations in documents [Grabmair et al., 2015]; (2) machine learning to manage complex non-structured information sets through machine learning techniques ("predictive coding") [Privault et al., 2010] and to recognize facts and discussions in legal cases [Wiltshire Jr et al., 2002]; (3) analysis of information from statutory texts, to classify paragraphs of statutory texts in terms of conceptual ontologies [Boella et al., 2012], to extract legal rules or norms [Wyner and Governatori, 2013], and to compare regulatory rules across jurisdictions [Savelka et al., 2014]; (4) extraction of arguments from cases, identifying the structure of arguments [Mochales Palau and Moens, 2011], the claims, and the substantive legal factors [Ashley and Brüninghaus, 2009]; (5) prediction of the outcome of judicial cases [Katz et al., 2017]. The current challenge is to integrate computational models for legal reasoning and ar- gumentation with machine learning approaches aimed at extracting the relevant knowledge [Ashley, 2017]. One of the most promising AI applications in the legal domain is the use of the IBM Watson technology to deliver QA services ${ }^{2}$, by extracting information from vast sets of legal documents, after being trained on the correlation between questions and answers.

\section{The Present: The Big Data Era and Risks for Consumers}

The character of legal scholarly reflection about AI has significantly shifted over the last five years, as a response to the wide-spread commercial success of AI implementations. Suddenly AI, often discussed jointly with big data [André et al., 2017], robotics [Balkin, 2015] and more generally "algorithms" [Gal and Elkin-Koren, 2017], came to be seen as a source of dangers and risks that need to be addressed by the law- and policy-maker [Scherer, 2015].

Several initiatives aiming to ensure that the development and use of AI will not pose substantial dangers to society and individuals have been launched by academia ${ }^{3}$, third sector ${ }^{4}$, governments [Cath et al., 2017] and business consortia ${ }^{5}$. Subsequently, a series of scholarly articles and monographs have been published. There are a few recurring general themes, including the questions of liability for actions of artificial agents [Vladeck, 2014], the legal status of artificial agents, particularly the question of granting them a sort of "digital personality" [Bryson et al., 2017], the inexplicability and non-transparency of decisions made by machine-learningpowered systems [Doshi-Velez et al., 2017], fairness [Helveston, 2015] data-bias and discrimination [Kim, 2017]. It also appears that the attention of legal academics has been attracted especially by some specific sectors, such as competition law [Ezrachi and Stucke, 2016], high-frequency trading of stocks [McNamara, 2016], self-driving cars [Schellekens, 2015] and smart cities [Eisen, 2013]. Among the suggested responses we find making software source code public, creating administrative agencies to oversee the AI development and deployment [Scherer, 2015], and granting the companies using AI particular fiduciary status [Balkin, 2017].

In our opinion, initiatives aimed at addressing "legal problems caused by AI in general" will keep growing, as a result of the snowball effect and the growing public interest in AI, but their added value will soon come to an end. AI is not a single technology; it includes many different approaches, that are used for a variety of purposes by a variety of actors. Consequently, the types of challenges caused by the use and deployment of AI will vary, and so will the regulatory responses they will prompt.

\footnotetext{
${ }^{2}$ See: ROSS Intelligence - Artificial Intelligence Meets Legal Research: https://rossintelligence.com/

${ }^{3}$ See, for example, the Ethics and Governance of Artificial Intelligence Fund at the Berkman Klein Center for Internet and Society at Harvard University and MIT Media Lab: https://cyber.harvard.edu/ research/ai

${ }^{4}$ See, for example, the AI initiative of the Future of Life Institute: https://futureoflife.org/ai-news/

${ }^{5}$ See, e.g.: Partnership on AI: https://www.partnershiponai.org/
} 
We believe that what is necessary right now is a sectoral, bottom-up approach, grounded in empirical research and particular fields of law, executed in an interdisciplinary dialogue of lawyers and engineers. Lawyers alone, being normative scholars, are well-trained to identify social challenges and propose regulatory goals, but without an ongoing exchange with engineers they risk talking not about what AI actually is and does, but rather their sci-fi driven idea of AI (e.g. machines will soon be conscious, and so they deserve a status of persons), and proposing solutions that are inoperable (e.g. making the source code public will increase accountability of big-data driven algorithms). In our current research [Micklitz et al., 2017; Lippi et al., 2017; Lippi et al., 2018] we focus on the challenges to European consumer law and policy stemming from the deployment of AI by businesses ${ }^{6}$.

European Union's consumer law has been created in order to address the factual inequality in the relations between consumers and business [Micklitz et al., 2017]. It contains, among others, provisions regarding advertisements, obligatory consumer rights, prohibited clauses in consumer contracts and information duties on the side of the business [Reich et al., 2014]. However, as the majority, if not all, branches of the legal system, it has been created before the massdeployment of AI, and thus it is not perfectly suited to address the more recent challenges brought about by it.

ARTSY $^{7}$ is an ongoing project where we analyze the use of AI by businesses through the lens of four general principles of consumer law. These are: the weaker party protection principle, privacy protection, the regulated autonomy principle, and the non-discrimination principle, as identified by Norbert Reich [2014]. After an empirical study of the spheres where business uses artificial intelligence, we narrowed down our focus to four sectors: advertising, data gathering, automated decision making and personal assistants. In our opinion, one should distinguish the new types of risks to consumers stemming from the use of AI made by business, such as the risk of replicating societal bias in advertising leading to discrimination [Sweeney, 2013], from the challenges to enforcers in oversight and detection, stemming both from the "blackbox" problem, and from background infringement happening inside computer systems, but invisible to the naked eye. The former beg for wise regulatory goals and invite normative considerations of new types of questions. For instance, should discrimination in advertising be prohibited? Should price discrimination be regulated by consumer law? Should profiling-based advertising be labelled as such? The latter beg for wise governance structures, leading to an incentive system that does not create chilling effects or impede innovation, but at the same time successfully addresses the challenges created by mass AI deployment. There might be situations where standards can help, but there might situations where, due to real-time flow of data, they just will not. Some business sectors, e.g. medical applications, might require ex-

\footnotetext{
${ }^{6}$ The final reports will be available in July 2018 at the website of the ARTSY project: https://artsy.eui.eu/

${ }^{7}$ ARTificial intelligence SYstems and consumer law and policy, see https://artsy.eui.eu/
}

ante control of the products before marketing them, while in case of others, e.g. recommendations-generating agents, it might be sufficient to control them ex-post. A wide range of available regulatory tools [Kroll et al., 2016] will need to be transformed into a nuanced and diverse regulatory environment, with different solutions to different problems, depending on the type of endangered legally-protected good (health, financial stability, non-discrimination etc.).

These challenges will need to be addressed by AIresponsive consumer law, not only regulating, but also facilitating fair development and use. Several initiatives have been put in place to debate these problems. However, we argue that the field of consumer law and AI should not only focus on addressing the challenges posed by AI. Presently, the mass deployment of $\mathrm{AI}$ is the domain of business, but this does not have to, and should not, remain this way. That is why, in the next section, we speak about the potential of AI to empower consumer and civil society, by creating tools that could help enforcers control business behaviour, and increase consumer knowledge, autonomy and ability to react.

\section{The Future: AI for Empowering Consumers}

As we witness the tremendous transformations in AI and its application domains, in AI and Law as well we discern the emergence of a growing phenomenon: the focus on the application and development of methods and tools for empowering consumers as a response to the power and technological supremacy of companies and business. This sort of "counterpower" yearned for by consumers is taking different shapes.

A first, straightforward application, is that of building systems capable of protecting consumers against unwanted monitoring and data collection, or against aggressive advertising. One popular instance of this problem is given by the adblocking and anti-adblocking battle [Nithyanand et al., 2016] between publishers and consumers: on one side, companies aim at developing tools for targeted advertising that exploit personal data of users, whereas consumers, on the other side, need instruments that safeguard their right to privacy [Jin, 2018]. Currently, there are proposals that try to spread compromise solutions, named privacy-friendly advertising [Guha et al., 2011], or frameworks that allow users to better control the ads and trackers they are exposed to [Yu et al., 2016]. The political world is becoming increasingly conscious of these demands, as it is confirmed by the new legislation adopted by the EU on the use of cookies. ${ }^{8}$

Similarly, big data mining techniques are more and more often used to extract patterns from the personal data of users, in order to support subsequent decision making processes [Athey, 2017; Pedreschi, 2017]. Typical examples are recommendation systems that suggest products to consumers, decision support systems in the banking and loan market aiming to profile and classify customers, and tracking tools for browsing content. Yet, in these scenarios, the use of automatic systems very often produces the risk of introducing user discrimination, in terms of race, gender, social or cultural status [Romei and Ruggieri, 2014]. In order to counter this negative effect, some classifiers have been proposed that can

\footnotetext{
${ }^{8}$ http://ec.europa.eu/ipg/basics/legal/cookies/
} 
enhance fairness and remove prejudice in data mining techniques [Kamishima et al., 2012]. Privacy-aware algorithms and techniques have been developed as well [Ruggieri et al., 2010]. Clearly, social networks represent the ideal ground for these kinds of applications, and for these reasons there is a growing interest in developing tools for personal data analysis in social networks [Rieder, 2013], even by large companies such as Wolfram Alpha for Facebook.

Another major aim of this "counter-power" paradigm shift will be that of building systems that co-enable consumers and their organizations to detect and contest unfair uses of $\mathrm{AI}$ and ICT, and in particular to support consumers in controlling the fairness of commercial practice. Some systems have been proposed, for example, to assess the trustworthiness and helpfulness of online consumer reviews by analyzing sentiment and polarity [Salehan and Kim, 2016; Villaroel Ordenes et al., 2016]. Other systems are devoted to the automatic detection of unfair clauses in online contracts, such as Terms of Services, using either rule-based systems built on grammars [Micklitz et al., 2017] or machine learning systems [Lippi et al., 2017]. Such tools have reached a considerable level of accuracy, and can be effectively used by consumer associations as well as the general public. For instance, in [Lippi et al., 2018] we show a publicly available online system, named CLAUDETTE, ${ }^{10}$ for detecting potentially unfair clauses in online terms of service with over $80 \%$ precision and recall. We are currently extending the system in order to cover data protection policy analysis as well.

As we discussed previously, AI-based technologies can also be used to develop tools for the efficient retrieval of information from huge data collections. Besides greatly improving the quality of work of professionals in the legal domain, such instruments could also be used by consumer associations as supporting platforms whose goal is to protect the interests of their affiliates. For example, the LawInsider platform ${ }^{11}$ provides a powerful search engine that manages over one million legal contracts, while the Evisort ${ }^{12}$ platform, based on cloud computing and AI techniques, provides tools for the management of, and information extraction from, legal documents such as contracts and provisions.

From a broader perspective, many other ICT sectors are openly supporting the development of technologies aimed to support customers and improve their quality of life. This is the case, for example, of the telecommunications industry within smart cities, where free connections that enable a variety of services to the citizen are becoming common. Massive Open Online Courses (MOOCs) are another example of a major technological effort to empower the people, not with smart services but with knowledge, and are helping disseminating knowledge and culture all around the world [Pappano, 2012]. A similar momentum has characterized the movement of Linked Open Data [Bizer et al., 2009], with a strong participation of politics and civil society. The legal domain still lags behind, possibly due to the presence of an initial knowledge

\footnotetext{
${ }^{9} \mathrm{http}: / / \mathrm{www}$. wolframalpha.com/facebook/

${ }^{10} \mathrm{http}: / /$ claudette.eui.eu

${ }^{11} \mathrm{https}: / /$ www.lawinsider.com/about

${ }^{12} \mathrm{http}: / /$ evisort.com/
}

barrier that is particularly hard to surmount. Like in other domains, encoding and digesting high-level knowledge represents a critical bottleneck, which must be solved before such knowledge can be put to good use and unlock services accessible to the wide public. We believe, however, that AI could help overcoming this barrier, by enabling tools and technologies that provide the public with information and knowledge that otherwise would be hard (or even impossible) for them to access.

We also believe that the AI community would benefit from a positive impact of consumer-empowering AI on how AI itself will be perceived by the public. One of the next big challenges, for example, is the debate on data privacy. Even as we write this article, the debate is prominent in the news, with Facebook under the spotlight and the US Congress rethinking their data privacy legislation. Europe has been at the forefront in data privacy law for a few years and reached a milestone in 2016, when the EU General Data Protection Regulation (GDPR), ${ }^{13}$ allegedly the most important change in data privacy regulation in 20 years, was approved by the EU Parliament. The GDPR is coming in force in May this year. Organizations are now in the process of changing the ways in which they process personal data, in order to comply with the new regulation, whose violations may lead to heavy fines. This involves changing the ways consumer data are collected, processed and transmitted to third parties, which will hopefully contribute to a fairer and more transparent interaction between companies and consumers. However, as soon as the milestone regulation is in place, the question remains of the extent to which companies will really comply with the letter and the spirit of the GDPR. Here is where AI can act as a novel democratizing force able to offer effectively consumerempowering tools.

Our CLAUDETTE project is currently studying a particular, but crucial aspect of compliance, fairness and transparency, namely, the drafting of privacy policies. Such policies are the documents through which data controllers inform users/consumers about the purposes and means of their personal data processing, and possibly ask for their consent (when consent provides the legal ground for the processing). The question to be addressed is then whether the new privacy policies will actually comply will the GDPR requirements concerning the information to be provided to consumers (data subjects), and how this information is to be presented. Effectiveness of enforcement is difficult, but crucial. How will individual citizens actually notice if privacy policies are unlawful or potentially misleading? Do consumer organizations have the resources required for following this up?

\section{Conclusions}

This work was motivated by two ambitions: an analytic one and a normative one. In the analytic strand, we discussed the transformation of the legal discourse about AI as a social and technological phenomenon. We showed that the discipline has existed for almost three decades now, with an initial strong focus on theoretical questions of knowledge representation and formalization of legal reasoning. The focus has

\footnotetext{
${ }^{13} \mathrm{https}: / /$ www.eugdpr.org
} 
indeed shifted about five years ago, when AI-powered applications started being widely used by businesses. AI as a subject made it to the mainstream of legal academia, is currently being discussed from a variety of angles, and catalyzes the attention of the public. However, the discursive frame has changed significantly. AI is currently seen as a source of threats and challenges, and as a phenomenon needing the attention of lawmakers and regulators. Notwithstanding the importance of these endeavours, we argue that legal scholars should not restrict themselves to combating the challenges posed by AI, but they should also embrace AI as a tool with a potential to empower civil society and the consumers. Certain initiatives in this strand can already be seen, though they still remain rather peripheral.

This is where our normative argument goes. We argued that applied AI does not have to remain in the hands of big businesses and corporations alone, but it can (and should) be brought to individual consumers, their organisations (civil society), as well as consumer agencies (public authorities), created to represent and defend consumer interests. We believe that the potential for this new direction is there. Some ongoing initiatives we surveyed seem to confirm that. However, this will not happen without a political effort, since one cannot expect the costs of such research to be funded by the commercial sector. Likewise, political action cannot be born in a vacuum, but it should be fostered by awareness. Sometimes, as it has been observed in other similar contexts, such as the movement for supporting net neutrality, it is thanks to initiatives arising from the population and the civil society that significant steps forward are made.

We thus envision consumer-empowering AI to act as a flywheel of change, by promoting awareness, and in a sense democratizing AI, while enabling bottom-up initiatives and incentivizing regulatory processes that would otherwise be hampered by an apparent imbalance between citizens, or even consumer organizations, and the big businesses holding data and technology.

\section{References}

[Allen and Saxon, 1994] Layman E. Allen and Charles S. Saxon. A-Hohfeld: A language for robust structural representation of knowledge in the legal domain to build interpretation-assistance expert systems. In John-Jules Ch. Meyer and Roel J. Wieringa, editors, Deontic Logic in Computer Science, pages 205-224. John Wiley and Sons Ltd., Chichester, UK, 1994.

[Allen and Widdison, 1996] Tom Allen and Robin Widdison. Can computers make contracts? Harvard Journal of Law \& Technology, 9:25, 1996.

[André et al., 2017] Quentin André, Ziv Carmon, Klaus Wertenbroch, Alia Crum, Douglas Frank, William Goldstein, Joel Huber, Leaf van Boven, Bernd Weber, and Haiyang Yang. Consumer choice and autonomy in the age of artificial intelligence and big data. Customer Needs and Solutions, pages 1-10, 2017.

[Article 29 Working Party, 2017] Article 29 Working Party. Guidelines on automated individual decision-making and profiling for the purposes of regulation 2016/679. 2017.
[Ashley and Brüninghaus, 2009] Kevin D Ashley and Stefanie Brüninghaus. Automatically classifying case texts and predicting outcomes. Artificial Intelligence and Law, 17(2):125-165, 2009.

[Ashley, 1991] Kevin D Ashley. Modeling legal arguments: Reasoning with cases and hypotheticals. MIT press, 1991.

[Ashley, 2017] Kevin D Ashley. Artificial Intelligence and Legal Analytics: New Tools for Law Practice in the Digital Age. Cambridge University Press, 2017.

[Athey, 2017] Susan Athey. Beyond prediction: Using big data for policy problems. Science, 355(6324):483-485, 2017.

[Balkin, 2015] Jack M Balkin. The path of robotics law. The Circuit, 6:45-60, 2015.

[Balkin, 2017] Jack M Balkin. The three laws of robotics in the age of big data. Ohio State Law Journal, 78, 2017. Yale Law School, Public Law Research Paper No. 592.

[Bizer et al., 2009] Christian Bizer, Tom Heath, and Tim Berners-Lee. Linked data - the story so far. International journal on semantic web and information systems, 5(3):122, 2009.

[Boella et al., 2012] Guido Boella, Luigi Di Caro, Leonardo Lesmo, and Daniele Rispoli. Multi-label classification of legislative text into EuroVoc. In Proceedings of the 25th Annual Conference Legal Knowledge and Information Systems, JURIX, volume 250 of Legal Knowledge and Information Systems, pages 21-30. IOS Press, 2012.

[Bryson et al., 2017] Joanna J Bryson, Mihailis E Diamantis, and Thomas D Grant. Of, for, and by the people: the legal lacuna of synthetic persons. Artificial Intelligence and Law, 25(3):273-291, 2017.

[Capper and Susskind, 1988] Phillip Capper and Richard E Susskind. Latent Damage Law: The Expert System. Butterworths, 1988.

[Cath et al., 2017] Corinne Cath, Sandra Wachter, Brent Mittelstadt, Mariarosaria Taddeo, and Luciano Floridi. Artificial intelligence and the 'good society': the US, EU, and UK approach. Science and engineering ethics, pages 1-24, 2017.

[Doshi-Velez et al., 2017] Finale Doshi-Velez, Mason Kortz, Ryan Budish, Chris Bavitz, Sam Gershman, David O'Brien, Stuart Schieber, James Waldo, David Weinberger, and Alexandra Wood. Accountability of AI under the law: The role of explanation. arXiv preprint arXiv:1711.01134, 2017.

[Eisen, 2013] Joel B Eisen. Smart regulation and federalism for the smart grid. Harvard Environmental Law Review, $37: 1,2013$.

[Ezrachi and Stucke, 2016] Ariel Ezrachi and Maurice E Stucke. Virtual competition. Oxford University Press, 2016.

[Gal and Elkin-Koren, 2017] Michal S Gal and Niva ElkinKoren. Algorithmic consumers. Harvard Journal of Law \& Technology, 30(2):309, 2017. 
[Gordon et al., 2007] Thomas F. Gordon, Henry Prakken, and Douglas N. Walton. The Carneades model of argument and burden of proof. Artificial Intelligence, 171:87596, 2007.

[Gordon et al., 2009] Thomas F Gordon, Guido Governatori, and Antonino Rotolo. Rules and norms: Requirements for rule interchange languages in the legal domain. In Rule interchange and applications, pages 282-296. Springer, 2009.

[Grabmair et al., 2015] Matthias Grabmair, Kevin D Ashley, Ran Chen, Preethi Sureshkumar, Chen Wang, Eric Nyberg, and Vern R Walker. Introducing LUIMA: an experiment in legal conceptual retrieval of vaccine injury decisions using a uima type system and tools. In Proceedings of the 15th International Conference on Artificial Intelligence and Law - ICAIL, pages 69-78. ACM, 2015.

[Guha et al., 2011] Saikat Guha, Bin Cheng, and Paul Francis. Privad: Practical privacy in online advertising. In USENIX conference on Networked systems design and implementation, pages 169-182, 2011.

[Helveston, 2015] Max N Helveston. Consumer protection in the age of big data. Washington University Law Review, 93:859, 2015

[Horty and Bench-Capon, 2012] John F. Horty and Trevor J. M. Bench-Capon. A factor-based definition of precedential constraint. Artificial intelligence and Law, 20:181214, 2012.

[Jin, 2018] Ginger Zhe Jin. Artificial intelligence and consumer privacy. In Economics of Artificial Intelligence. University of Chicago Press, 2018.

[Kamishima et al., 2012] Toshihiro Kamishima, Shotaro Akaho, Hideki Asoh, and Jun Sakuma. Fairness-aware classifier with prejudice remover regularizer. In Proceedings of the 2012 European Conference on Machine Learning and Knowledge Discovery in Databases Volume Part II, ECML PKDD'12, pages 35-50, Berlin, Heidelberg, 2012. Springer-Verlag.

[Katz et al., 2017] Daniel Martin Katz, Michael J Bommarito II, and Josh Blackman. A general approach for predicting the behavior of the supreme court of the united states. PloS one, 12(4):e0174698, 2017.

[Kim, 2017] Pauline T Kim. Auditing algorithms for discrimination. University of Pennsylvania Law Review Online, 166(1):10, 2017.

[Kroll et al., 2016] Joshua A Kroll, Solon Barocas, Edward W Felten, Joel R Reidenberg, David G Robinson, and Harlan Yu. Accountable algorithms. University of Pennsylvania Law Review, 165:633, 2016.

[Lippi et al., 2017] Marco Lippi, Przemysław Pałka, Giuseppe Contissa, Francesca Lagioia, Hans-Wolfgang Micklitz, Yannis Panagis, Giovanni Sartor, and Paolo Torroni. Automated detection of unfair clauses in online consumer contracts. In Proceedings of the 30th Annual Conference on Legal Knowledge and Information Systems, JURIX, volume 302 of Frontiers in Artificial Intelligence and Applications, pages 145-154. IOS Press, 2017.
[Lippi et al., 2018] Marco Lippi, Przemysław Pałka, Giuseppe Contissa, Francesca Lagioia, Hans-Wolfgang Micklitz, Giovanni Sartor, and Paolo Torroni. CLAUDETTE: an automated detector of potentially unfair clauses in online terms of service. arXiv preprint arXiv:1805.01217, 2018.

[McNamara, 2016] Steven McNamara. The law and ethics of high-frequency trading. Minnesota Journal of Law, Science \& Technology, 17:71, 2016.

[Micklitz et al., 2017] Hans-W Micklitz, Przemysław Pałka, and Yannis Panagis. The empire strikes back: Digital control of unfair terms of online services. Journal of Consumer Policy, 40(3):367-388, 2017.

[Mochales Palau and Moens, 2011] Raquel Mochales Palau and Marie-Francine Moens. Argumentation mining. Artificial Intelligence and Law, 19(1):1-22, 2011.

[Nithyanand et al., 2016] Rishab Nithyanand, Sheharbano Khattak, Mobin Javed, Narseo Vallina-Rodriguez, Marjan Falahrastegar, Julia E. Powles, Emiliano De Cristofaro, Hamed Haddadi, and Steven J. Murdoch. Adblocking and counter blocking: A slice of the arms race. In Amir Houmansadr and Prateek Mittal, editors, 6th USENIX Workshop on Free and Open Communications on the Internet, FOCI '16, Austin, TX, August 8, 2016. USENIX Association, 2016.

[O’Neil, 2016] Cathy O’Neil. Weapons of Math Destruction: How Big Data Increases Inequality and Threatens Democracy. Crown Publishing Group, New York, NY, USA, 2016.

[Pappano, 2012] Laura Pappano. The year of the MOOC. The New York Times, 2(12):2012, 2012.

[Pedreschi, 2017] Dino Pedreschi. Social network analytics, data science ethics \& privacy-preserving analytics. In 1st Europe Summer School: Data Science, SummerSchool '17, New York, NY, USA, 2017. ACM.

[Prakken and Sartor, 1998] Henry Prakken and Giovanni Sartor. Modelling reasoning with precedents in a formal dialogue game. Artificial Intelligence and Law, 6:231-87, 1998.

[Prakken and Sartor, 2015] Henry Prakken and Giovanni Sartor. Law and logic: A review from an argumentation perspective. Artificial Intelligence, 227:214-45, 2015.

[Prakken et al., 2015] Henry Prakken, Adam Wyner, Trevor Bench-Capon, and Katie Atkinson. A formalisation of argumentation schemes for legal case-based reasoning in ASPIC+. Journal of Logic and Computation, 25:11411166, 2015.

[Prakken, 2010] Henry Prakken. An abstract framework for argumentation with structured arguments. Argument and Computation, 1:93-124, 2010.

[Privault et al., 2010] Caroline Privault, Jacki O’Neill, Victor Ciriza, and Jean-Michel Renders. A new tangible user interface for machine learning document review. Artificial Intelligence and Law, 18(4):459-479, 2010. 
[Reich et al., 2014] Norbert Reich, Hans-Wolfgang Micklitz, Peter Rott, and Klaus Tonner. European consumer law. Intersentia, 2014.

[Reich, 2014] Norbert Reich. General principles of EU civil law. Intersentia, 2014.

[Rieder, 2013] Bernhard Rieder. Studying Facebook via data extraction: the Netvizz application. In Proceedings of the 5th annual ACM web science conference, pages 346-355. ACM, 2013.

[Romei and Ruggieri, 2014] Andrea Romei and Salvatore Ruggieri. A multidisciplinary survey on discrimination analysis. The Knowledge Engineering Review, 29(5):582638, 2014.

[Ruggieri et al., 2010] Salvatore Ruggieri, Dino Pedreschi, and Franco Turini. Data mining for discrimination discovery. ACM Transactions on Knowledge Discovery from Data, 4(2):9:1-9:40, May 2010.

[Salehan and Kim, 2016] Mohammad Salehan and Dan J. Kim. Predicting the performance of online consumer reviews: A sentiment mining approach to big data analytics. Decision Support Systems, 81:30 - 40, 2016.

[Sartor, 2009] Giovanni Sartor. Cognitive automata and the law: electronic contracting and the intentionality of software agents. Artificial Intelligence and Law, 17(4):253290, 2009.

[Savelka et al., 2014] Jaromír Savelka, Matthias Grabmair, and Kevin D Ashley. Mining information from statutory texts in multi-jurisdictional settings. In Proceedings of the 27th Annual Conference Legal Knowledge and Information Systems, JURIX, volume 271 of Legal Knowledge and Information Systems, pages 133-142, 2014.

[Schellekens, 2015] Maurice Schellekens. Self-driving cars and the chilling effect of liability law. Computer Law \& Security Review, 31(4):506-517, 2015.

[Scherer, 2015] Matthew U Scherer. Regulating artificial intelligence systems: Risks, challenges, competencies, and strategies. Harvard Journal of Law \& Technology, 29:353, 2015.

[Sweeney, 2013] Latanya Sweeney. Discrimination in online ad delivery. arXiv preprint arXiv:1301.6822, 2013.

[Verheij, 2003] Bart Verheij. Artificial argument assistants for defeasible argumentation. Artificial Intelligence, 150(1-2):291-324, 2003.

[Villaroel Ordenes et al., 2016] F Villaroel Ordenes, S Ludwig, D Grewal, K de Ruyter, and M Wetzels. Analyzing online reviews through the lens of speech act theory: implications for consumer sentiment analysis. Journal of Consumer Research, 2016.

[Vladeck, 2014] David C Vladeck. Machines without principals: liability rules and artificial intelligence. Washington Law Review, 89:117, 2014.

[Wagstaff, 2012] Kiri Wagstaff. Machine learning that matters. In Proceedings of the 29th International Conference on Machine Learning, ICML, Edinburgh, Scotland, UK, June 26 - July 1, 2012. icml.cc / Omnipress, 2012.
[Wiltshire Jr et al., 2002] James S Wiltshire Jr, John T Morelock, Timothy L Humphrey, X Allan Lu, James M Peck, and Salahuddin Ahmed. System and method for classifying legal concepts using legal topic scheme, December 31 2002. US Patent 6,502,081.

[Wyner and Governatori, 2013] Adam Z. Wyner and Guido Governatori. A study on translating regulatory rules from natural language to defeasible logics. In Joint Proceedings of the 7th International Rule Challenge, the Special Track on Human Language Technology and the 3rd RuleML Doctoral Consortium, Seattle, USA, July 11 13, 2013, volume 1004 of CEUR Workshop Proceedings. CEUR-WS.org, 2013.

[Yu et al., 2016] Zhonghao Yu, Sam Macbeth, Konark Modi, and Josep M Pujol. Tracking the trackers. In Proceedings of the 25th International Conference on World Wide Web, pages 121-132. International World Wide Web Conferences Steering Committee, 2016. 